Supporting information

\title{
Ultra-stretchable polyaniline-based conductive organogel with high strain sensitivity
}

Yucheng Zhang ${ }^{1}$, Yusen Zhao ${ }^{1}$, Zihang Peng ${ }^{1}$, Bowen Yao ${ }^{1,2}$, Yousif Alsaid ${ }^{1}$, Mutian Hua ${ }^{1}$, Dong

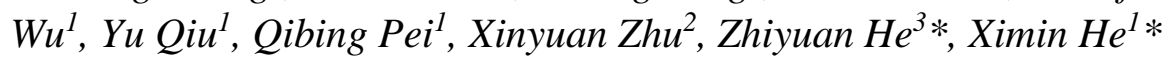

Y. Zhang, Y. Zhao, Z. Peng, Dr. B. Yao, Y. Alsaid, M. Hua, D. Wu, Y. Qiu, Prof. Q. Pei, Prof. X. He

Department of Materials Science and Engineering, University of California, Los Angeles, CA 90095, USA

B. Yao, Prof. X. Zhu

School of Chemistry and Chemical Engineering, State Key Laboratory of Metal Matrix

Composites, Shanghai Jiao Tong University, Shanghai 200240, China.

Prof. Z. He

Institute of Chemistry, University of Chinese Academy of Sciences, 100190 Beijing, China

Corresponding Authors: Prof. X. He, E-mail: ximinhe@ucla.edu; Prof. Z. He, E-mail:

hezy@iccas.ac.cn 


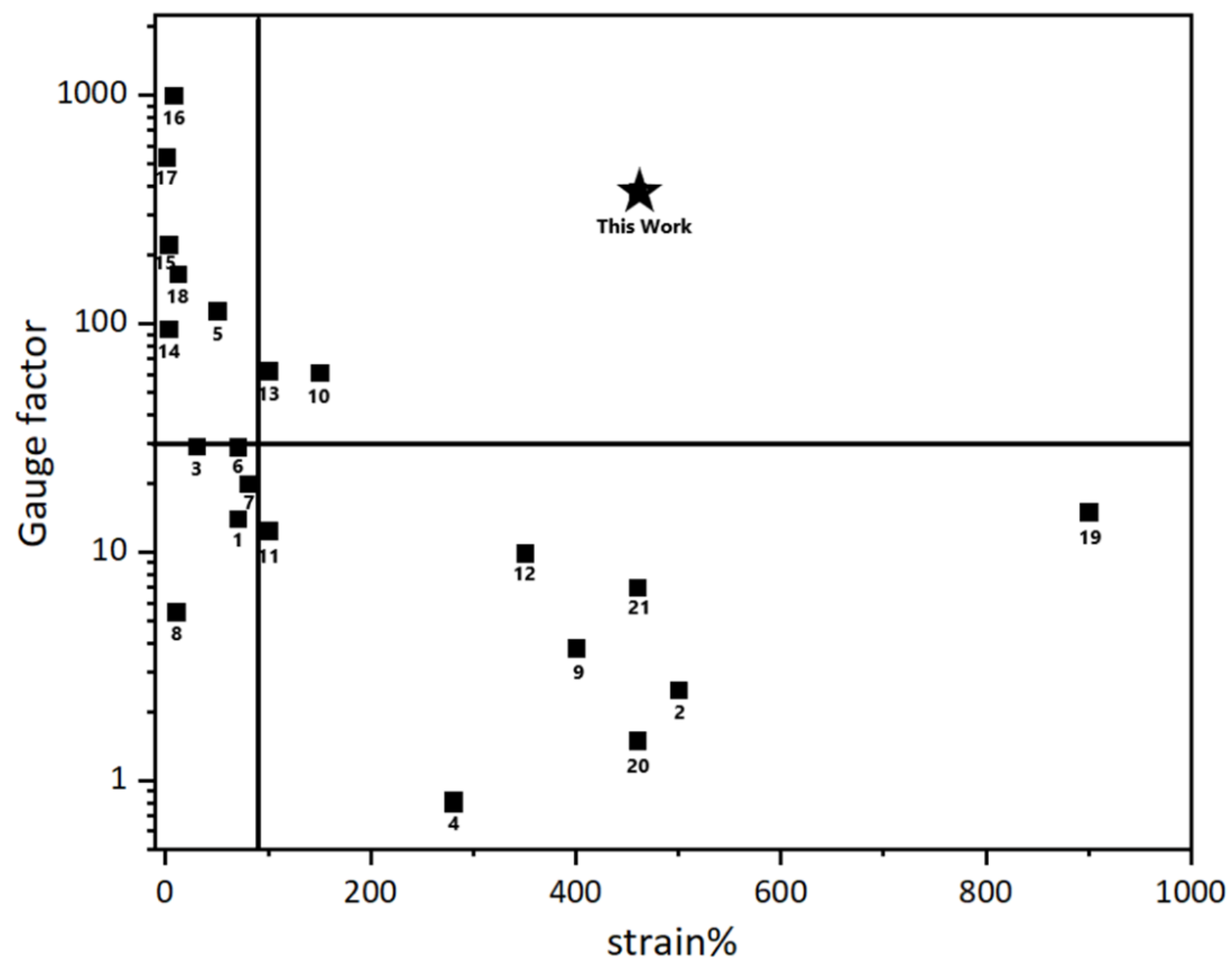

Figure S1 Systematic summary of GF/strain in strain sensors. *These reference publications are mainly selected from film structure strain sensors, other high sensitivity from fibers or special structures are not included.

Reference

[1]M. Amjadi, A. Pichitpajongkit, S. Lee, S. Ryu , I. Park, ACS Nano 2014, 8, 5154.

[2]M. Amjadi, Y. J. Yoon, I. Park, Nanotechnology 2015, 26, 375501.

[3]N. Lu, C. Lu, S. Yang, J. Rogers, Adv. Funct. Mater. 2012, 22, 4044.

[4]T. Yamada, Y. Hayamizu, Y. Yamamoto, Y. Yomogida, A. Izadi-Najafabadi, D. N. Futaba, K. Hata, Nat. Nanotechnol. 2011, 6, 296.

[5] X. Xiao, L. Yuan, J. Zhong, T. Ding, Y. Liu, Z. Cai, Y. Rong, H. Han, J. Zhou, Z. L. Wang, Adv. Mater. 2011, 23,54400 .

[6] Y. R. Jeong, H. Park, S. W. Jin, S. Y. Hong, S. S. Lee, J. S. Ha, Adv. Funct. Mater. 2015, 25, 4228.

[7]C. Mattmann, F. Clemens, G. Tröster, Sensors 2008, 8, 3719. 
[8] J. Kong, N. Jang, S. Kim, J. Kim, Carbon 2014, 77, 199.

[9] J. Muth, D. M. Vogt, R. Truby , Y. Mengüç, D. B. Kolesky , R. J. Wood , J. A. Lewis , Adv. Mater. 2014, $26,6307$.

[10] S. Gong, D. Lai, Y. Wang, L. W. Yap, K. J. Si, Q. Shi, N. N. Jason, T. Sridhar, H. Uddin, W. Cheng, ACS Appl. Mater. Interfaces 2015, 7, 19700.

[11] B.-U. Hwang, J.-H. Lee , T. Q. Trung , E. Roh , D.-I. Kim , S.-W. Kim , N.-E. Lee , ACS Nano 2015 , 9 , 8801 .

[12] S. Gong, D. T. Lai , B. Su , K. J. Si , Z. Ma , L. W. Yap , P. Guo , W. Cheng , Adv. Electron. Mater. 2015, $1,1400063$.

[13] E. Roh, B.-U. Hwang , D. Kim , B.-Y. Kim , N.-E. Lee , ACS Nano 2015 , 9 , 6252.

[14] K. Takei, Z. B. Yu, M. Zheng, H. Ota, T. Takahashi, A. Javey, Proc. Natl. Acad. Sci. USA 111 (5) (2014) 1703-1707.

[15] X. Liu, C. Tang, X. H. Du, S. Xiong, S. Y. Xi, Y. F. Liu, et al., Mater. Horiz. 4 (3) (2017) 477-486.

[16] Q. Liu, M. Zhang, L. Huang, Y. R. Li, J. Chen, C. Li, et al., ACS Nano 9 (12) (2015) 12320-12326.

[17] X. Q. Liao, Q. L. Liao, X. Q. Yan, Q. J. Liang, H. N. Si, M. H. Li, et al., Adv. Funct. Mater. 25 (16) (2015) 2395-2401.

[18] G. Shi, Z. H. Zhao, J. H. Pai, I. Lee, L. Q. Zhang, C. Stevenson, et al., Adv. Funct. Mater. 26 (42) (2016) 7614-7625.

[19] S. Lee, S. Shin, S. Lee, J. Seo, J. Lee, S. Son, et al., Adv. Funct. Mater. 25 (21) (2015) 3114-3121.

[20] P. Lee, J. Lee, H. Lee, J. Yeo, S. Hong, K. H. Nam, et al., Adv. Mater. 24 (25) (2012) 3326-3332.

[21] P. Lee, J. Ham, J. Lee, S. Hong, S. Han, Y. D. Suh, et al., Adv. Funct. Mater. 24 (36) (2014) 5671-5678. 


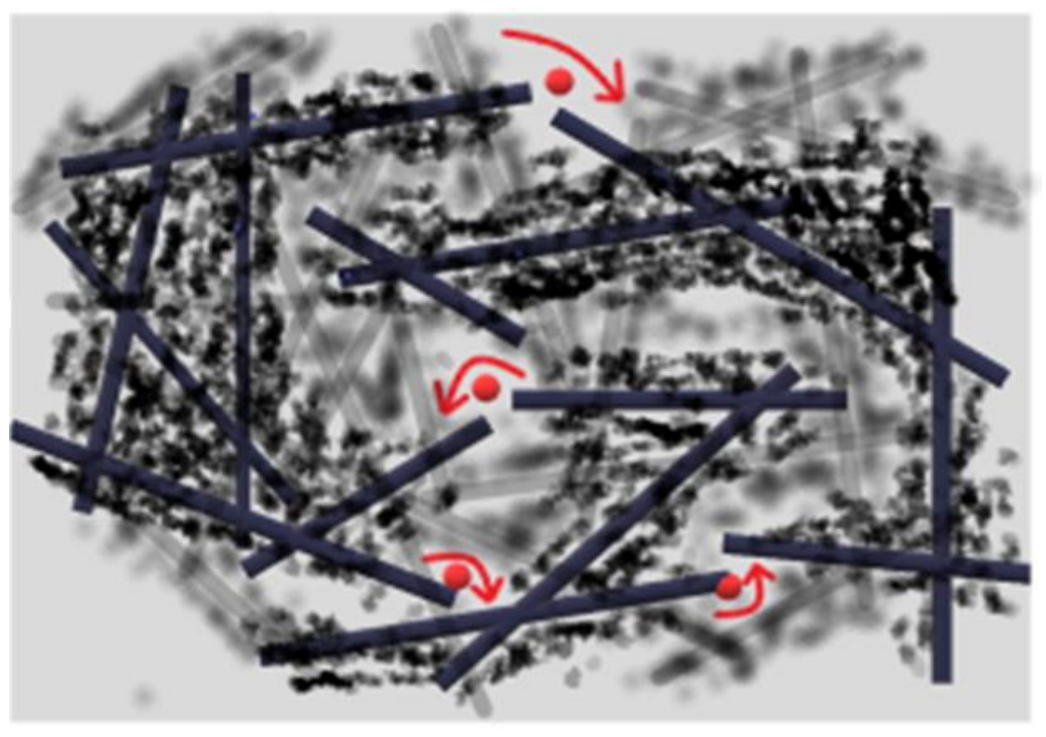

Figure S2. Tunneling effect schematic diagram. Black and grey rods and dots indicate polyaniline blend layer, red dots with arrow represent electrons hopping.

The tunneling resistivity of Polyaniline blend is estimated as

$$
R_{\text {tunneling }}=\frac{V}{A J}=\frac{h^{2} d}{A e^{2} \sqrt{2 m \lambda}} \exp \left(\frac{4 \pi d}{h} \sqrt{2 m \lambda}\right)
$$

, where $\mathrm{J}$ is tunneling current density, $\mathrm{V}$ is the electrical potential difference, $\mathrm{e}$ is the single electron charge, $m$ is the mass of electron, $h$ is Planck's constant, $d$ is the distance between NWs, $\lambda$ is the height of the energy barrier and $A$ is the cross-sectional area of the tunnel.

\section{Reference}

[1] M. Amjadi, A. Pichitpajongkit, S. Lee, S. Ryu , I. Park, ACS Nano 2014, 8, 5154. 


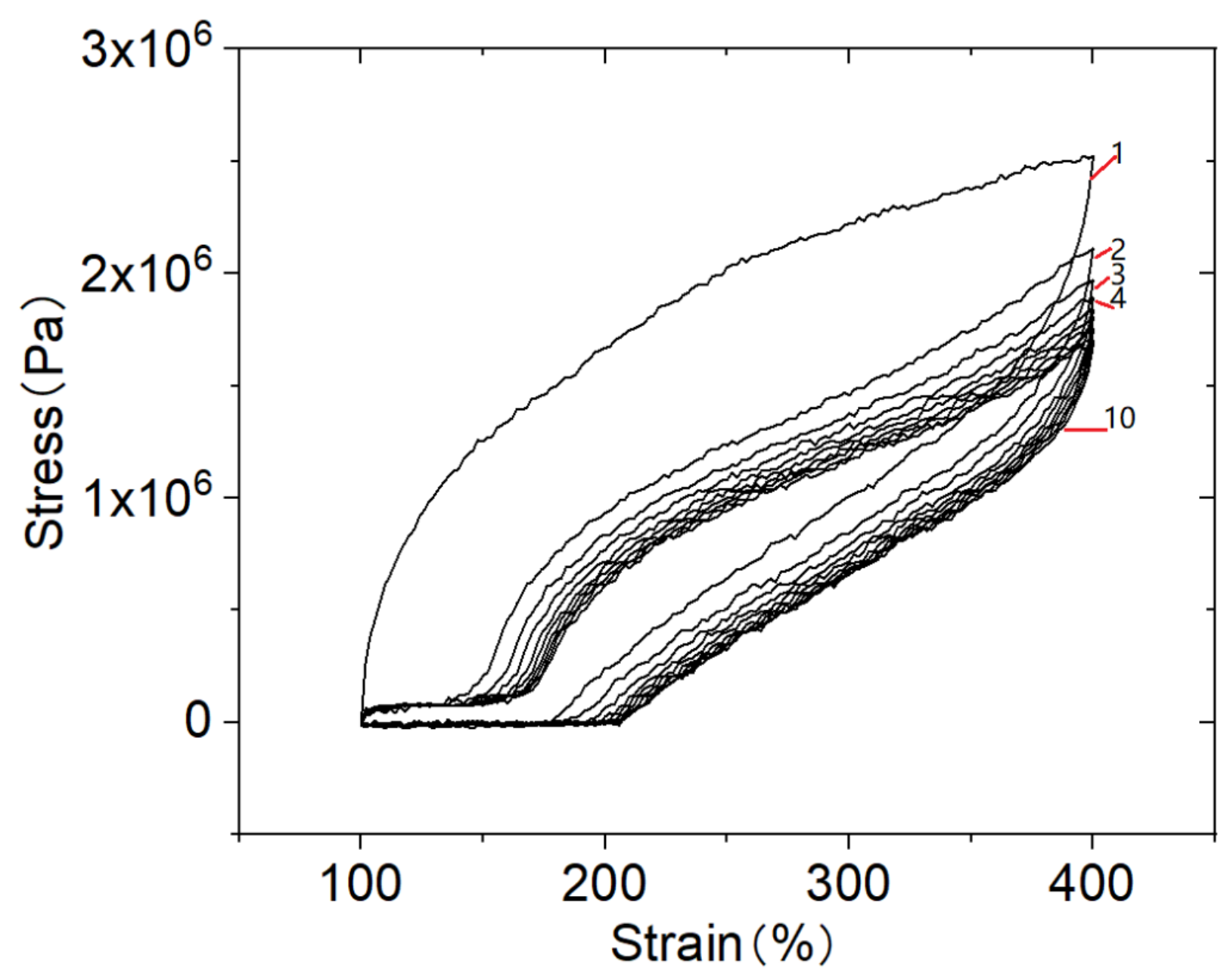

Figure S3. Repetitive stress-strain test on polymethyl acrylate-PANi organogel with a maximum strain at $400 \%$ 


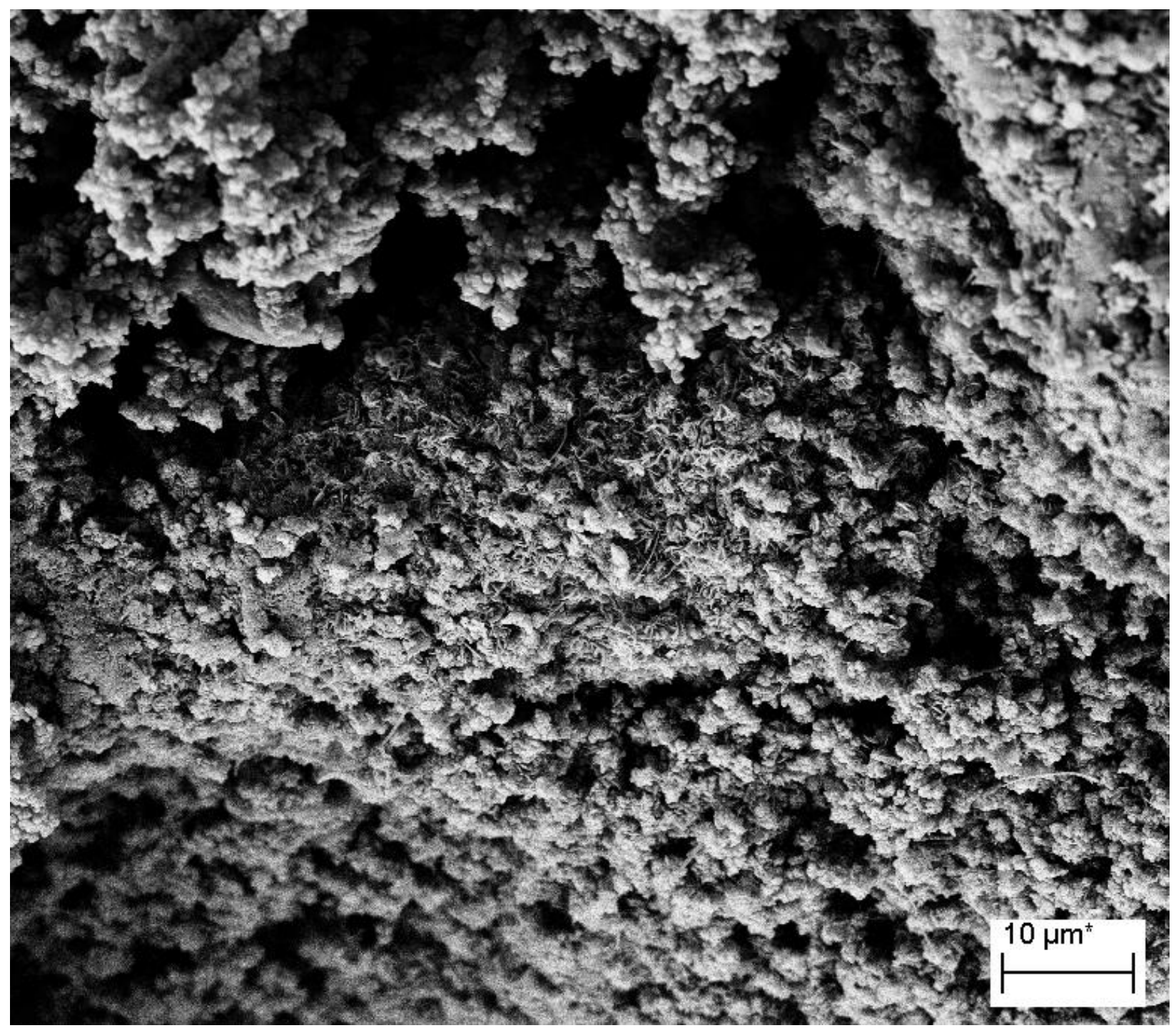

Figure S4. SEM figure on the PANi blend structure. The morphology of the surface conductive PANi are mainly clusters and smaller rods underneath. 

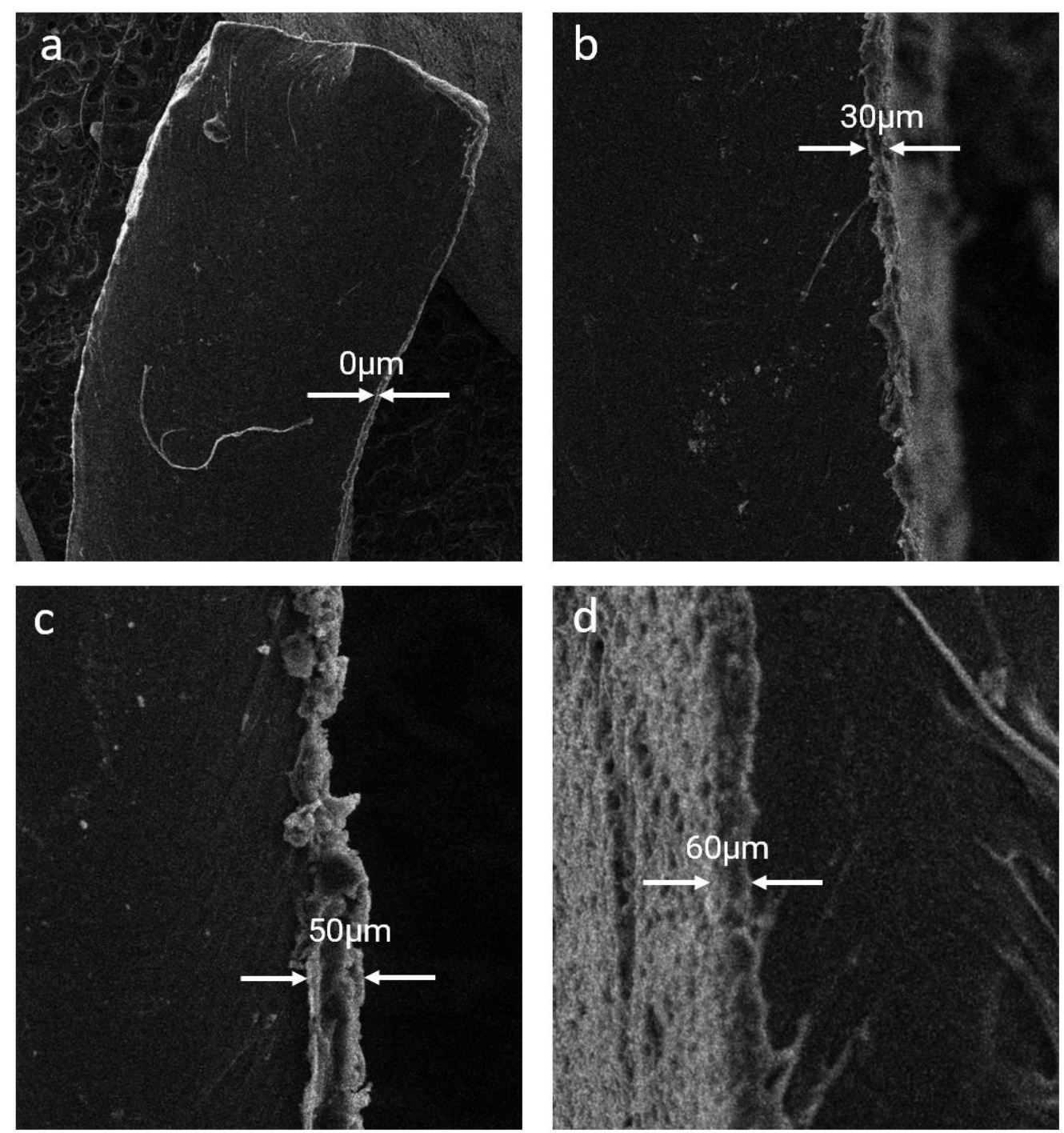

Figure S5. SEM images of the polyaniline/polymethyl acrylate organogels, with different aniline ratios as shown in the table below. The polyaniline layer thickness is indicated within each figure. All the samples have the same size.

\begin{tabular}{|c|c|c|c|c|}
\hline & Methyl acrylate $(\mathrm{ml})$ & DMF $(\mathrm{ml})$ & Aniline $(\mathrm{ml})$ & Aniline ratio (\%) \\
\hline $\mathrm{a}$ & 1.2 & 0.80 & 0.00 & 0.0 \\
\hline $\mathrm{b}$ & 1.2 & 0.71 & 0.09 & 4.6 \\
\hline $\mathrm{c}$ & 1.2 & 0.62 & 0.18 & 9.1 \\
\hline $\mathrm{d}$ & 1.2 & 0.53 & 0.27 & 13.7 \\
\hline
\end{tabular}




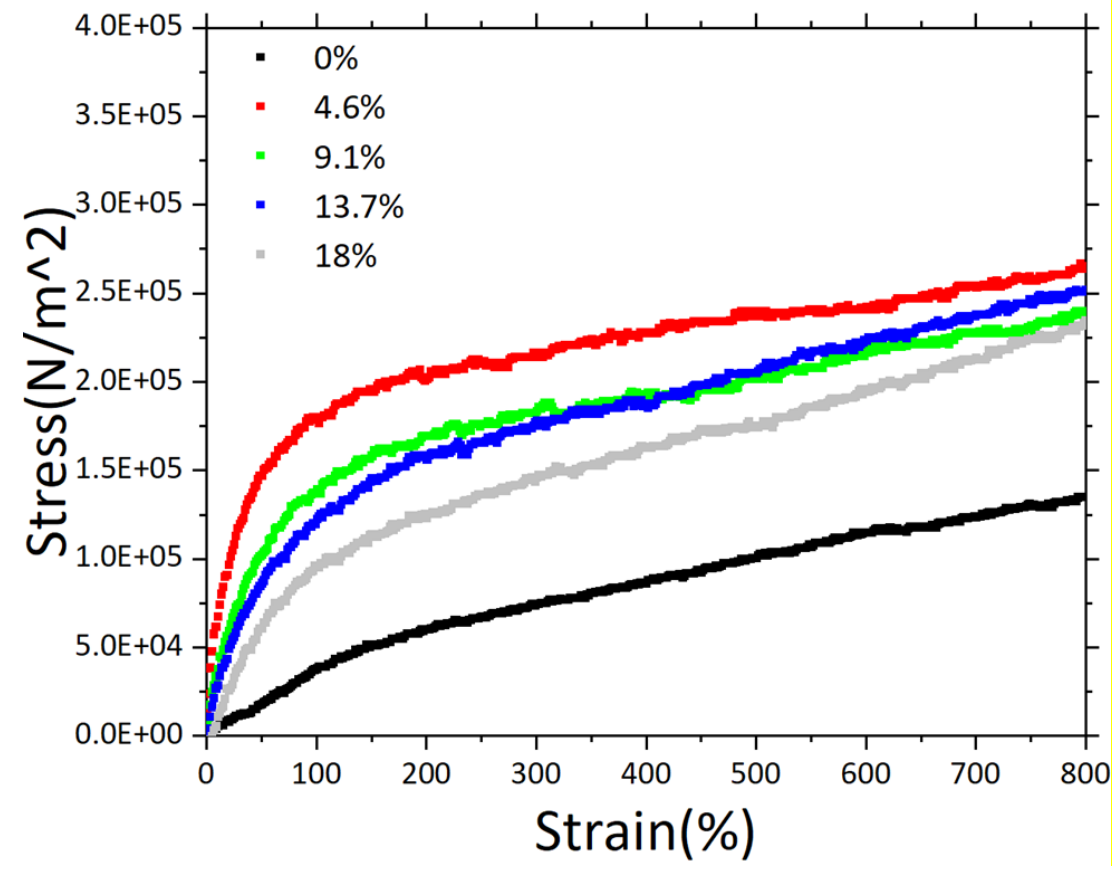

Figure S6. The stress-strain curves of PANi/polymethyl acrylate organogels with different aniline ratios from $0 \%$ to $18 \%$. 